\title{
RECURSIVE EQUIVALENCE TYPES AND OCTAHEDRA
}

\author{
J. C. E. DEKKER \\ (Received 12 March 1981, revised 9 March 1982) \\ Communicated by J. N. Crossley
}

\begin{abstract}
Let the word "graph" be used in the sense of a countable, connected, simple graph with at least one vertex. We write $Q_{n}$ and $\mathrm{Oc}_{n}$ for the graphs associated with the $n$-cube $Q^{n}$ and the $n$-octahedron $\mathrm{Oc}^{n}$ respectively. In a previous paper (Dekker, 1981) we generalized $Q_{n}$ and $Q^{n}$ to a graph $Q_{N}$ and a cube $Q^{N}$, for any nonzero recursive equivalence type $N$. In the present paper we do the same for $O c_{n}$ and $\mathrm{Oc}^{n}$. We also examine the nature of the duality between $Q^{N}$ and $\mathrm{Oc}^{N}$, in case $N$ is an infinite isol. There are $c$ RETs, $c$ denoting the cardinality of the continuum.
\end{abstract}

1980 Mathematics subject classification (Amer. Math. Soc.): primary 03 D 50; secondary 05 C 99.

\section{Preliminaries}

This paper is closely related to 'RETs and cubes', Dekker (1981), to which the reader is referred for notations and terminology not explained below. Propositions of that paper are referred to as P1.1, P1.2, .,P2.1, P2.2, ., and so on. We write $\varepsilon$ for the set $(0,1, \ldots)$, o for the empty set, $\Lambda$ for the collection of all isols and $\Omega$ for the collection of all RETs. Also, $\varepsilon_{0}=\varepsilon-(0), \Lambda_{0}=\Lambda-(0)$ and $\Omega_{0}=\Omega-(0)$. We again use the canonical enumeration $\left\langle\rho_{n}\right\rangle$ of the class of all finite sets, that is, finite subsets of $\varepsilon$. For a finite set $\sigma$ the unique number $i$ such that $\sigma=\rho_{i}$ is the canonical index of $\sigma$, written $\operatorname{can}(\sigma)$. Put $r_{n}=$ card $\rho_{n}$; for $\nu \subset \varepsilon$, $i \in \varepsilon$ we define $2^{\nu}=\left\{x \in \varepsilon \mid \rho_{x} \subset \nu\right\}$ and $[\nu ; i]=\left\{x \in \varepsilon \mid \rho_{x} \subset \nu \& r_{x}=i\right\}$. These recursive, combinatorial operators enable us to extend the functions $2^{n}$ and $[n ; i]$ from $\varepsilon$ into $\varepsilon$ to functions $2^{N}$ and $[N ; i]$ from $\Omega$ into $\Omega$. The ordinary, vertical notations for the binomial functions $[n ; i]$ and $[N ; i]$ are only used in displayed

Copyright Australian Mathematical Society 1983 
formulas. If $f$ is a function from a subset of $\varepsilon$ into $\varepsilon$, we denote its domain by $\delta f$, its range by $\rho f$ and the image of $n$ under $f$ by $f(n)$ or $f_{n}$, sometimes in the same context. Throughout this paper the symbols $\nu, \nu_{0}, \nu_{1}, \nu_{2}$ denote nonempty sets, while $\mu, \mu_{0}, \mu_{1}, \mu_{2}$ stand for sets of cardinality $\geqslant 2$.

We define the (undirected) $\omega$-cube $Q^{\nu}$ on the set $\nu$ as the ordered pair $\left\langle 2^{\nu}, F_{\nu}\right\rangle$, where $F_{\nu}$ is the class of all faces of $Q^{\nu}$, that is, the class of all finite subsets $\sigma$ of $2^{\nu}$ for which there exist disjoint finite subsets $\rho_{p}$ and $\rho_{q}$ of $\nu$ such that $\sigma=\left\{x \in 2^{\nu} \mid\right.$ $\left.\rho_{p} \subset \rho_{x} \subset \rho_{p} \cup \rho_{q}\right\}$. The number $j(p, q)$ is the G-number $G(\sigma)$ of $\sigma$ and the number $k=r_{q}$ the dimension of $\sigma$; we refer to $\sigma$ as a $k$-face of $Q^{\nu}$ and write $F_{\nu k}$ for the class of all $k$-faces of $Q^{\nu}$. All faces of $Q^{\nu}$ are therefore finite-dimensional, even if the $\operatorname{RET} N=\operatorname{Req} \nu$, the so-called $\omega$-dimension of $Q^{\nu}$, is infinite, that is, belongs to $\Omega-\varepsilon$. We use the term " $N$-cube" for an $\omega$-cube of $\omega$-dimension $N$. For $k \in \varepsilon$, $N \subset \Omega_{0}, \nu \in N$ we define

$$
\begin{aligned}
\alpha_{\nu k}=\left\{G(\sigma) \in \varepsilon \mid \sigma \in F_{\nu k}\right\}, & \alpha_{\nu}=\left\{G(\sigma) \in \varepsilon \mid \sigma \in F_{\nu}\right\}, \\
A_{N k}=\operatorname{Req} \alpha_{\nu k}, & A_{n}=\operatorname{Req} \alpha_{\nu} .
\end{aligned}
$$

The functions $A_{N k}$ and $A_{N}$ are well-defined, that is, independent of the representative $\nu$ of $N$. According to P4.1 and P4.2 we have for $N \in \Omega_{0}$ and $0 \leqslant k \leqslant N$,

$$
A_{N k}=\left(\begin{array}{l}
N \\
k
\end{array}\right) 2^{N-k}, \quad A_{n}=3^{N} .
$$

\section{Octahedral graphs}

The six vertices and twelve edges of a regular octahedron in $E^{3}$ form a graph; its vertices can be denoted by $1, \ldots, 6$ so that the pairs of opposite vertices are $(1,2),(3,4),(5,6)$; it is therefore isomorphic to the complete tripartite graph $\mathrm{O}_{3}=K(2,2,2)$ with two vertices in each partite set. For $n \geqslant 1$ the $n$-octahedral graph $O_{n}$ is defined as the $n$-partite graph $K(2, \ldots, 2)$ with two vertices in each of its $n$ partite sets, Jungerman and Ringel (1978). Let $O_{n}$ have $\mu=(1, \ldots, 2 n)$ as set of vertices and $((1,2), \ldots,(2 n-1,2 n))$ as class of its partite sets. Define $f$ as the permutation of $\mu$ which interchanges $2 k-1$ and $2 k$, for $1 \leqslant k \leqslant n$. We now drop the condition that $\mu=(1, \ldots, 2 n)$ and define an involution without fixed points (iwfp) of $\mu$ as a permutation $f$ of $\mu$ such that $f^{2}=i_{\mu}$ and $f(x) \neq x$, for $x \in \mu$. Denote the family of all iwfps of $\mu$ by $\operatorname{Inv}(\mu)$, associate with every $f \in \operatorname{Inv}(\mu)$ the graph $G_{f}=\langle\mu, \theta\rangle$, where $\theta=\{\operatorname{can}(x, y) \in[\mu ; 2] \mid f(x) \neq y\}$, and call a (countable) graph $G=\langle\mu, \theta\rangle$ octahedral, if $G=G_{f}$, for some $f \in \operatorname{Inv}(\mu)$. Note that $f \rightarrow G_{f}$ maps $\operatorname{Inv}(\mu)$ one-to-one onto the family of all octahedral graphs with $\mu$ as set of vertices. The vertices $p$ and $q$ of $G_{f}$ are opposite, if $f(p)=q$ or equivalently, $f(q)=p$; thus $p$ and $q$ are adjacent iff they are not opposite. The 
iwfp $f$ of $\mu$ is an $\omega$-iwfp of $\mu$, if it has a partial recursive one-to-one extension; here "one-to-one" can be deleted without changing the concept defined, since $f=f^{-1}$. Denote the family of all $\omega$-iwfps of $\mu$ by $\operatorname{Inv}_{\omega}(\mu)$. If $G=\langle\mu, \theta\rangle$ we call the function $f$ such that $G=G_{f}$ the octahedral function of $G$; the graph $G$ is $\omega$-octahedral, if its octahedral function belongs to $\operatorname{Inv}_{\omega}(\mu)$. We claim that in case $f \in \operatorname{Inv}(\mu)$, we have $: f \in \operatorname{Inv}_{\omega}(\mu)$ if and only if $f$ is the restriction to $\mu$ of some partial recursive iwfp of some r.e. superset of $\mu$. For if $\bar{f}$ is a partial recursive extension of $f$ we put $\bar{\mu}=\left\{x \in \delta \bar{f} \mid \bar{f}(x) \neq x \& \bar{f}(x) \in \delta \bar{f} \& \bar{f}^{2}(x)=x\right\}$; then $\bar{\mu}$ is r.e., $\mu \subset \bar{\mu}$ and $\bar{f} \mid \bar{\mu}$ is a partial recursive extension of $f$. Note that $\operatorname{Inv}_{\omega} \mu=\operatorname{Inv} \mu$, if $\mu$ is finite. Thus a finite graph is $\omega$-octahedral if and only if it is octahedral.

Proposition B2.1. Let $M=\operatorname{Req} \mu$. Then there is an w-octahedral graph with $\mu$ as set of vertices if and only if $M$ is even.

Proof. Let $G=\langle\mu, \theta\rangle$ and $M=\operatorname{Req} \mu$. Suppose $G$ is $\omega$-octahedral, say $G=G_{f}$, for $f \in \operatorname{Inv}_{\omega}(\mu)$. Then $f=\bar{f} \mid \mu$, for some partial recursive iwfp $\bar{f}$ of some r.e. superset of $\mu$, say $\bar{\alpha}$. Define

$$
\begin{cases}\bar{\alpha}_{0}=\{x \in \bar{\alpha} \mid x<\bar{f}(x)\}, & \bar{\alpha}_{1}=\{x \in \bar{\alpha} \mid x>\bar{f}(x)\}, \\ \alpha_{0}=\bar{\alpha}_{0} \cap \mu, \quad \alpha_{1}=\bar{\alpha}_{1} \cap \mu, \quad \delta \bar{g}=\bar{\alpha}_{0}, \quad \bar{g}=\bar{f}\left|\bar{\alpha}_{0}, \quad g=f\right| \alpha_{0},\end{cases}
$$

then $\bar{g}$ is a partial recursive one-to-one function from $\bar{\alpha}_{0}$ onto $\bar{\alpha}_{1}$ which maps $\alpha_{0}$ onto $\alpha_{1}$, hence $\alpha_{0} \simeq \alpha_{1}$. Clearly, $\mu=\alpha_{0} \cup \alpha_{1}$, where $\alpha_{0} \mid \alpha_{1}$, hence $M=2 \operatorname{Req} \alpha_{0}$ is even. Now assume that $M=\operatorname{Req} \mu$ is even, say $\mu=\alpha_{0} \cup \alpha_{1}, \alpha_{0} \simeq \alpha_{1}$ and $\alpha_{0} \mid \alpha_{1}$. Let $g$ be a one-to-one function from $\alpha_{0}$ onto $\alpha_{1}$ with a partial recursive one-to-one extension. Put $\delta f=\mu, f(x)=g(x)$, for $x \in \alpha_{0}$ and $f(x)=g^{-1}(x)$, for $x \in \alpha_{1}$. Then $f$ is an $\omega$-iwfp of $\mu$ and $G_{f}$ is an $\omega$-octahedral graph with $\mu$ as set of vertices.

REMARK. Since $\Lambda-\varepsilon$ has cardinality $c$ and the mappings $X \rightarrow 2 X$ and $X \rightarrow$ $2 X+1$ are one-to-one, there are exactly $c$ infinite even isols and exactly $c$ infinite odd isols. Thus we see by B2.1 that there are exactly $c \omega$-octahedral graphs. With every infinite odd isol $M$ we can associate a set $\mu \in M$ and (since $\boldsymbol{\kappa}_{0}=2 \boldsymbol{\kappa}_{0}$ ) an iwfp $f$ of $\mu$ and the octahedral graph $G_{f}$. In view of B2.1 the graph $G_{f}$ is not $\omega$-octahedral. It follows that there also are exactly $c$ octahedral graphs which are not $\omega$-octahedral.

The graph $G=\langle\nu, \eta\rangle$ is $r . e$., if the sets $\nu$ and $\eta$ are r.e. We call a connected graph $G$ an $\omega$-graph, if it has an MPA (minimal path algorithm), that is, if there is an effective procedure II which associates with every two distinct vertices of $G$ a path of minimal length between them. An $\omega$-graph $G=\langle\nu, \eta\rangle$ is uniform, if $G$ is an induced subgraph of some r.e. $\omega$-graph $\bar{G}$ which has an MPA $\bar{\Pi}$ which when 
applied to distinct vertices $p$ and $q$ of $G$ yields a minimal path between $p$ and $q$ in $G$, that is, a minimal path with vertices in $\nu$ and edges in $\eta$. It was proved by Remmel (1981) that an $\omega$-graph $G=\langle\nu, \eta\rangle$ need not be uniform, even if $\nu$ is immune.

Proposition B2.2. Let $G=\langle\mu, \theta\rangle$ be an $\omega$-octahedral graph. Then $G$ is a uniform $\omega$-graph and there is a nonzero RET $N$ such that $\operatorname{Req} \mu=2 N$ and $\operatorname{Req} \theta=2 N(N-1)$.

Proof. Let $G=\langle\mu, \theta\rangle$, say $G=G_{f}$, for $f \in \operatorname{Inv}_{\omega}(\mu)$. Define $\alpha_{0}$ and $\alpha_{1}$ as in (2.1), then $M=\operatorname{Req} \mu=2 N$, where $N=\operatorname{Req} \alpha_{0}$. Put

$$
\lambda=\left\{\operatorname{can}(x, y) \in[\mu ; 2] \mid x \in \alpha_{0} \& y \in \alpha_{1} \& f(x) \neq y\right\},
$$

then Req $\lambda=N(N-1)$ and $\theta=\left[\mu_{0} ; 2\right] \cup\left[\mu_{1} ; 2\right] \cup \lambda$, where the three sets on the right are separable. Hence Req $\theta$ equals $2[N ; 2]+\operatorname{Req} \lambda$, that is, $2 N(N-1)$. Note that this proof is valid both in case $N \in \Lambda_{0}$, that is, if $N-1<N$ and in case $N \in \Omega_{0}-\Lambda$, that is, if $N-1=N$. In the latter case $\operatorname{Req} \theta=2 N^{2}$. We now prove that $G_{f}=\langle\mu, \theta\rangle$ is a uniform $\omega$-graph. Let $f=\bar{f} \mid \mu$, where $\bar{f}$ is a partial recursive iwfp of a r.e. superset of $\mu$, say $\bar{\alpha}$. Define $\bar{\alpha}_{0}, \bar{\alpha}_{1}, \alpha_{0}, \alpha_{1}$ as in (2.1) and $\bar{\theta}=\{\operatorname{can}(x, y) \in[\bar{\alpha} ; 2] \mid \bar{f}(x) \neq y\}$, then $G_{f}$ is an induced subgraph of the r.e. graph $\bar{G}=\langle\bar{\alpha}, \bar{\theta}\rangle$. We may assume without loss of generality that card $\alpha_{0} \geqslant 2$. Let $p, u \in \alpha_{0}, p \neq u, q=f(p)$, then $p, q, u$ are distinct vertices of $G$ and $p, q$ are opposite. Put for $x, y \in \bar{\alpha}, x \neq y$,

$$
\bar{\pi}(x, y)= \begin{cases}\langle x, u, y\rangle, & \text { if } x=p \text { and } y=q, \text { or } x=q \text { and } y=p, \\ \langle x, p, y\rangle, & \text { if } x \notin(p, q), \text { but } \bar{f}(x)=y, \\ \langle x, y\rangle, & \text { if } \bar{f}(x) \neq y .\end{cases}
$$

Then $\bar{\pi}_{x y}$ is a minimal path between $x$ and $y$ in $\bar{G}$ which is a minimal path in $G$ in case $x, y \in \mu$. Since $\bar{\pi}_{x y}$ can be effectively obtained from $x$ and $y$, the $\omega$-graph $G$ is uniform.

Define the functions $d_{0}, d_{1}$ by $\delta d_{0}=\delta d_{1}=\varepsilon, d_{0}(x)=2 x, d_{1}(x)=2 x+1$ and associate with every set $\nu$ the sets $\nu_{0}=d_{0}(\nu)$ and $\nu_{1}=d_{1}(\nu)$. The standard $\omega$-iwfp associated with $\nu$ is the function $f \in \operatorname{Inv}_{\omega}(\mu)$, where $\mu=\nu_{0} \cup \nu_{1}$ and $f(2 x)=2 x+1, f(2 x+1)=2 x$, for $x \in \nu$. The standard $\omega$-octahedral graph Oc associated with $\nu$ is the graph $G_{f}=\langle\mu, \theta\rangle$, where $f$ is the standard $\omega$-iwfp associated with $\nu$. Thus the vertices $p$ and $q$ of $\mathrm{Oc}_{v}$ are opposite, if $p$ is even and $p+1=q$, or $q$ is even and $q+1=p$. An $\omega$-isomorphism from the graph $G_{1}$ onto the graph $G_{2}$ is an isomorphism from $G_{1}$ onto $G_{2}$ which has a partial recursive one-to-one extension. $G_{1}$ is isomorphic ( $\omega$-isomorphic) to $G_{2}$, if there is at least one 
isomorphism ( $\omega$-isomorphism) from $G_{1}$ onto $G_{2}$. These two equivalence relations are denoted by $\cong$ and $\cong \omega$. Two finite graphs are $\omega$-isomorphic if and only if they are isomorphic. Under an $\omega$-isomorphism from $G_{1}$ onto $G_{2}$ minimal paths in $G_{1}$ correspond to minimal paths in $G_{2}$. This implies

$$
\text { if } G_{1} \cong_{\omega} G_{2} \text { and } G_{1} \text { is an } \omega \text {-graph, so is } G_{2} \text {. }
$$

Proposition B2.3. A graph is $\omega$-octahedral if and only if it is $\omega$-isomorphic to some standard $\omega$-octahedral graph.

Proof. Let $G=\langle\mu, \theta\rangle$. (a) Suppose $g$ is an $\omega$-isomorphism from $\mathrm{Oc}_{\nu}=\left\langle\nu_{0} \cup\right.$ $\left.\nu_{1}, \eta\right\rangle$ onto $G$; put $\delta t=\nu_{0} \cup \nu_{1}, t(x)=x+1$, for $x \in \nu_{0}, t(x)=x-1$, for $x \in \nu_{1}$; then $\mathrm{Oc}_{\nu}=G_{t}$. Define $\delta f=\mu$, and $f=g \operatorname{tg}^{-1}$, then $f \in \operatorname{Inv}_{\omega}(\mu)$. Moreover, $\operatorname{can}(x, y) \in \theta$ if and only if $\operatorname{can}\left(g^{-1}(x), g^{-1}(y)\right) \in \eta$ if and only if $\operatorname{tg}^{-1}(x)=$ $g^{-1}(y)$ if and only if $f(x) \neq y$, so that $\theta=\{\operatorname{can}(x, y) \in[\mu ; 2] \mid f(x) \neq y\}$ and $G=G_{f}$. The function $t$ has a recursive one-to-one extension, namely $\bar{t}$, where $\delta \bar{t}=\varepsilon, \bar{t}(x)=x+1$, for $x \in \delta_{0}$, while $\bar{t}(x)=x-1$, for $x \in \delta_{1}$. Let $\bar{g}$ be a partial recursive one-to-one extension of $g$; put $\delta \bar{f}=\{x \in \rho \bar{g} \mid \bar{t}(x) \in \delta \bar{g}\}$ and $\bar{f}(x)=\bar{g} t \bar{g}^{-1}(x)$, then $\bar{f}$ is a partial recursive one-to-one extension of $f$, hence $G=G_{f}$ is $\omega$-octahedral.

(b) Assume that $G=\langle\mu, \theta\rangle$ is $\omega$-octahedral, say $G=G_{f}$, where $f \in \operatorname{Inv}_{\omega}(\mu)$. If $\mu$ is finite, put $m=\frac{1}{2}$ card $\mu, \nu=(0, \ldots, m-1)$; then $\mathrm{Oc}_{\nu} \cong G$, hence $\mathrm{Oc}_{\nu} \cong_{\omega} G$. Now assume that $\mu$ is infinite. Let $\bar{f}$ be a partial recursive iwfp of some r.e. superset of $\mu$, say $\bar{\mu}$, then there is a one-to-one recursive function $\bar{u}_{n}$ ranging over $\bar{\mu}$ such that $\bar{f}\left(\bar{u}_{2 n}\right)=\bar{u}_{2 n+1}$ and $\bar{u}_{2 n}<\bar{u}_{2 n+1}$. Then $\bar{u}_{2 n} \in \mu$ if and only if $\bar{u}_{2 n+1} \in \mu$, for $n \in \varepsilon$. Let $\nu=\left\{n \in \varepsilon \mid \bar{u}_{2 n} \in \mu\right\}$, then $\bar{u}\left(\nu_{0} \cup \nu_{1}\right)=\mu$. Define $\delta t=\nu_{0} \cup \nu_{1}, t(x)=x+1$, for $x \in \nu_{0}, t(x)=x-1$, for $x \in \nu_{1}$, then $\mathrm{Oc}_{v}=G_{t}$. For $\mathrm{Oc}_{\nu}=\left\langle\nu_{0} \cup \nu_{1}, \eta\right\rangle$ and $x, y \in \nu_{0} \cup \nu_{1}$,

$$
\begin{aligned}
\operatorname{can}(x, y) & \in \eta \Leftrightarrow t(x) \neq y \Leftrightarrow \bar{u}_{t(x)} \neq \bar{u}_{t(y)} \\
& \Leftrightarrow f\left(\bar{u}_{x}\right) \neq \bar{u}_{y} \Leftrightarrow \operatorname{can}\left(\bar{u}_{x}, \bar{u}_{y}\right) \in \theta .
\end{aligned}
$$

Thus $\bar{u} \mid \nu_{0} \cup \nu_{1}$ is an isomorphism from $\mathrm{Oc}_{\nu}$ onto $G$ with the recursive one-to-one extension $\bar{u}$, hence $\mathrm{Oc}_{\nu} \cong{ }_{\omega} G$.

If $G=\langle\mu, \theta\rangle$ is an $\omega$-graph, $o(G)=\operatorname{Req} \mu$ is the order of $G$. Thus $o(G)$ has the usual meaning if and only if $G$ is finite. If the graph $G=\langle\mu, \theta\rangle$ is $\omega$-octahedral, its order $M$ is even by $\mathrm{B} 2.1$, that is, $M=2 A$, for some $A \in \Omega$. We call $A$ the $\omega$-dimension of $G$, written $\operatorname{dim}_{\omega} G$. Since $2 A=2 B$ implies $A=B$ by Friedberg (1961), $\operatorname{dim}_{\omega} G$ is well-defined for an $\omega$-octahedral graph $G$. Hence two $\omega$-octahedral graphs have the same order if and only if they have the same $\omega$-dimension. 
PROPOSITION B2.4. Two w-octahedral graphs are w-isomorphic if and only if they have the same $\omega$-dimension.

Proof. Since $\operatorname{dim}_{\omega} \mathrm{Oc}_{\nu}=\operatorname{Req} \nu$, it suffices to show in view of B2.3 that $\alpha \simeq \beta \Leftrightarrow \mathrm{Oc}_{\alpha} \cong{ }_{\omega} \mathrm{Oc}_{\beta}$, for nonempty sets $\alpha$ and $\beta$. The conditional from the right to the left is trivial, since $\omega$-isomorphic graphs have the same order. Now assume $\alpha \simeq \beta$, say $\alpha \subset \delta p, p(\alpha)=\beta, p$ partial recursive and one-to-one, $\mathrm{Oc}_{\alpha}=\left\langle\alpha_{0} \cup\right.$ $\left.\alpha_{1}, \theta_{\alpha}\right\rangle, \mathrm{Oc}_{\beta}=\left\langle\beta_{0} \cup \beta_{1}, \theta_{\beta}\right\rangle$. Put $\delta q=d_{0}(\delta p) \cup d_{1}(\delta p), q(x)=2 p(x / 2)$, for $x \in \delta q \cap \delta_{0}$, while $q(x)=2 p(x-1 / 2)$, for $x \in \delta q \cap \delta_{1}$. Then $\alpha_{0} \cup \alpha_{1} \subset \delta q$, $q\left(\alpha_{0} \cup \alpha_{1}\right)=\beta_{0} \cup \beta_{1}$, where $q$ is partial recursive and one-to-one. Moreover, $\operatorname{can}(x, y) \in \theta_{\alpha}$ implies $\operatorname{can}\left(q_{x}, q_{y}\right) \in \theta_{\beta}$, for $x, y \in \alpha_{0} \cup \alpha_{1}$, so that $q \mid \alpha_{0} \cup \alpha_{1}$ is an $\omega$-isomorphism from $\mathrm{Oc}_{\alpha}$ onto $\mathrm{Oc}_{\beta}$.

For $N \in \Omega_{0}$ we define $\mathrm{Oc}_{N}$ as any $\omega$-octahedral graph of $\omega$-dimension $N$, or equivalently, of order $2 N$. Thus $\mathrm{Oc}_{N}$ is unique up to $\omega$-isomorphism. For the definitions of an $\omega$-regular graph and its $\omega$-degree, see page 546 of Dekker (1981a). It can be shown that every $\omega$-octahedral graph of order $2 N$ is $\omega$-regular of $\omega$-degree $2(N-1)$; the proof is routine.

\section{Octahedra}

With every $f \in \operatorname{Inv}(\mu)$ we associate the ordered pair $\mathrm{Oc}_{f}=\left\langle\mu, C_{\mu}\right\rangle$, where $\mu=\delta f$ and $C_{\mu}$ is the class of all finite subsets $\sigma$ of $\mu$ such that no two elements of $\sigma$ correspond to each other under $f$. An octahedron is an ordered pair Oc $=\left\langle\mu, C_{\mu}\right\rangle$ such that $\mathrm{Oc}=\mathrm{Oc}_{f}$, for some $f \in \operatorname{Inv}(\mu)$; $\mathrm{Oc}_{f}$ is an $\omega$-octahedron, if $f \in \operatorname{Inv}_{\omega}(\mu)$. The mapping $f \rightarrow \mathrm{Oc}_{f}$ maps $\operatorname{Inv}(\mu)$ one-to-one onto the family of all octahedra on $\mu$ and $\operatorname{Inv}_{\omega}(\mu)$ one-to-one onto the family of all $\omega$-octahedra on $\mu$. If $\mathrm{Oc}_{f}=\left\langle\mu, C_{\mu}\right\rangle$, then $G_{f}=\langle\mu, \theta\rangle$ where $\theta=\left\{\operatorname{can}(x, y) \in[\mu ; 2] \mid(x, y) \in C_{\mu}\right\}$, hence $\mathrm{Oc}_{f}$ and $G_{f}$ uniquely determine each other; we say that $\mathrm{Oc}_{f}$ and $G_{f}$ are associated. The members of $\mu$ are the vertices of $\mathrm{Oc}_{f}=\left\langle\mu, C_{\mu}\right\rangle$, while the members of $C_{\mu}$ are the faces of $\mathrm{Oc}_{f}$. If $\sigma$ is a face we define $\operatorname{dim} \sigma$ as the number $k=\operatorname{card} \sigma-1$ and refer to $\sigma$ as a $k$-face of $\mathrm{Oc}_{f}$; we write $C_{\mu k}$ for the class of all $k$-faces of $\mathrm{Oc}_{f}$. Note that (i) every face of $\left\langle\mu, C_{\mu}\right\rangle$ is finite-dimensional, even if $\mu$ is infinite, (ii) every subset of a face is again a face, (iii) there is only one $(-1)$-face, namely the empty set, (iv) $\mu \notin C_{\mu}$, since our agreement that card $\mu \geqslant 2$ implies that $\mu$ contains two opposite vertices. For the $\omega$-octahedron Oc $=\left\langle\mu, C_{\mu}\right\rangle$ with $M=$ Req $\mu$, we define the order $o(\mathrm{Oc})$ as $M$ and the $\omega$-dimension $\operatorname{dim}_{\omega}(\mathrm{Oc})$ as $M / 2$. Hence $o(\mathrm{Oc})=o(G)$ and $\operatorname{dim}_{\omega}(\mathrm{Oc})=\operatorname{dim}_{\omega}(G)$, where $G$ is associated with Oc. An isomorphism ( $\omega$-isomorphism) from $\mathrm{Oc}_{1}=\left\langle\mu_{1}, C_{\mu(1)}\right\rangle$ onto $\mathrm{Oc}_{2}=\left\langle\mu_{2}, C_{\mu(2)}\right\rangle$ is a one-to-one 
function (with a partial recursive one-to-one extension) from $\mu_{1}$ onto $\mu_{2}$ which preserves faces and their dimensions. We write $\cong$ for "isomorphic to" and $\cong$ for " $\omega$-isomorphic to." If $G_{1}$ and $G_{2}$ are associated with $\mathrm{Oc}_{1}$ and $O \mathrm{c}_{2}$ respectively, the isomorphisms ( $\omega$-isomorphisms) from $G_{1}$ onto $G_{2}$ are the same as the isomorphisms ( $\omega$-isomorphisms) from $\mathrm{Oc}_{1}$ onto $\mathrm{Oc}_{2}$. We have therefore by $\mathrm{B} 2.4$ for $\omega$-octahedra $\mathrm{Oc}_{1}=\left\langle\mu_{1}, C_{\mu(1)}\right\rangle$ and $\mathrm{Oc}_{2}=\left\langle\mu_{2}, C_{\mu(2)}\right\rangle$,

$$
\mu_{1} \simeq \mu_{2} \Leftrightarrow O c_{1} \cong{ }_{\omega} \mathrm{Oc}_{2} \Leftrightarrow \operatorname{dim}_{\omega} \mathrm{Oc}_{1}=\operatorname{dim}_{\omega} \mathrm{Oc}_{2} .
$$

The standard $\omega$-octahedron $\mathrm{Oc}^{\nu}$ associated with the set $\nu$ is the $\omega$-octahedron $\mathrm{Oc}_{f}$, where $f$ is the standard $\omega$-iwfp associated with $\nu$. In view of B2.3 and (3.1) we conclude that (i) an octahedron is an $\omega$-octahedron if and only if it is $\omega$-isomorphic to a standard $\omega$-octahedron, (ii) $\alpha \simeq \beta \Leftrightarrow \mathrm{Oc}^{\alpha} \cong{ }_{\omega} \mathrm{Oc}^{\beta}$, for $\alpha, \beta \neq o$. If $N \in \Omega_{0}$ we define $\mathrm{Oc}^{N}$ as any $\omega$-octahedron of $\omega$-dimension $N$; it is unique up to $\omega$-isomorphism. Let for $n \geqslant 1,-1 \leqslant k \leqslant n-1$, Oc ${ }^{n}$ have $c_{n k} k$-faces and $c_{n}$ faces. It is well-known and readily seen that $c_{n k}=2^{n+1}[n ; k+1]$ and $c_{n}=3^{n}$. In order to generalize these formulas to $\mathrm{Oc}^{N}$, for $N \in \Omega_{0}$, we define for $\mathrm{Oc}^{\nu}=$ $\left\langle\mu, C_{\mu}\right\rangle,-1 \leqslant k \leqslant N-1$,

$$
\begin{gathered}
\tilde{\gamma}_{\nu k}=\left\{x \in \varepsilon \mid \rho_{x} \in C_{\mu k}\right\}, \quad \tilde{\gamma}_{\nu}=\left\{x \in \varepsilon \mid \rho_{x} \in C_{\mu}\right\}, \\
C_{N k}=\operatorname{Req} \tilde{\gamma}_{\nu k}, \quad \text { for } \nu \in N, \quad C_{N}=\operatorname{Req} \tilde{\gamma}_{\nu}, \quad \text { for } \nu \in N, \\
\gamma_{\nu k}=\left\{j(p, q) \in \varepsilon \mid \rho_{p}, \rho_{q} \subset \nu \& \rho_{p} \cap \rho_{q}=o \& r_{p+q}=k+1\right\}, \\
\gamma_{\nu}=\left\{j(p, q) \in \varepsilon \mid \rho_{p}, \rho_{q} \subset \nu \& \rho_{p} \cap \rho_{q}=o\right\} .
\end{gathered}
$$

The functions $C_{N k}$ and $C_{N}$ are well-defined, that is, independent of the choice of $\nu$. Note that $x \in \tilde{\gamma}_{\nu k}$ if and only if $d_{0}^{-1}\left(\rho_{x} \cap \delta_{0}\right)$ and $d_{1}^{-1}\left(\rho_{x} \cap \delta_{1}\right)$ are disjoint subsets of $\nu$ and $r_{x}=k+1$. The $G$-number $G\left(\rho_{x}\right)$ of the face $\rho_{x}$ of $\operatorname{Oc}^{\nu}=\left\langle\mu, C_{\mu}\right\rangle$ is defined as $j(p, q)$, where $\rho_{p}=d_{0}^{-1}\left(\rho_{x} \cap \delta_{0}\right), \rho_{q}=d_{1}^{-1}\left(\rho_{x} \cap \delta_{1}\right)$. It can now be proved that

$$
\tilde{\gamma}_{\nu k} \simeq \gamma_{\nu k} \text { and } \quad \tilde{\gamma}_{\nu} \simeq \gamma_{\nu}, \quad \text { for }-1 \leqslant k \leqslant N-1, N \geqslant 1, \nu \in N .
$$

Proposition B3.1. For $N \in \Omega_{0}$ and $-1 \leqslant k \leqslant N-1$,

$$
C_{N k}=2^{k+1}\left(\begin{array}{c}
N \\
k+1
\end{array}\right) \text { and } C_{N}=3^{N}
$$

Proof. Let $\nu \in N$. Then we have $\gamma_{\nu} \simeq \alpha_{v}$ and $C_{N}=A_{N}=3^{N}$ by (1.1), (1.3), and (3.6). The formula for $C_{N k}$ is trivial for $k=-1$. Now assume $k \geqslant 0$. Put $\alpha=(0, \ldots, k)$ and $\beta=j\left[2^{\alpha} \times[\nu ; k+1]\right]$ then $\operatorname{Req} \beta=2^{k+1}[N ; k+1]$ and it suffices to prove $\beta \simeq \gamma_{\nu k}$. Let $\delta g=\beta$ and for $j(x, y) \in \beta, g j(x, y)=j(p, q)$, where $p$ and $q$ are computed as follows: find the enumeration according to size of $\rho_{y}$, say $z_{0}, \ldots, z_{k}$; then $\rho_{p}=\left\{z_{i} \mid 0 \leqslant i \leqslant k \& i \in \rho_{x}\right\}$ and $\rho_{q}=\rho_{y}-\rho_{p}$. Then $\rho_{p}$ and $\rho_{q}$ are disjoint subsets of $\nu$, while $r_{p+q}=r_{y}=k+1$, hence $j(p, q) \in \gamma_{\nu k}$, so 
that $g(\beta) \subset \gamma_{\nu k}$. It can now be proved that $g(\beta)=\gamma_{\nu k}$, where $g$ is one-to-one and both $g$ and $g^{-1}$ have partial recursive extensions. Thus $\beta \simeq \gamma_{\nu k}$.

\section{Some sequences of isols}

This section deals with the sequences defined by the functions $[n ; k]$, for $0 \leqslant k \leqslant n, \quad a_{n k}=2^{n-k}[n ; k]$ and $c_{n k}=2^{k+1}[n ; k+1]$, for $0 \leqslant k \leqslant n-1$, namely

$$
\begin{aligned}
& \text { I }\left\langle\left(\begin{array}{l}
n \\
k
\end{array}\right)\right\rangle_{k=0}^{n}:\left(\begin{array}{l}
n \\
0
\end{array}\right),\left(\begin{array}{l}
n \\
1
\end{array}\right), \ldots,\left(\begin{array}{c}
n \\
n-1
\end{array}\right),\left(\begin{array}{l}
n \\
n
\end{array}\right), \\
& \text { II }\left\langle a_{n k}\right\rangle_{k=0}^{n-1}: 2^{n}, 2^{n-1}\left(\begin{array}{c}
n \\
1
\end{array}\right), \ldots, 2^{2}\left(\begin{array}{c}
n \\
n-2
\end{array}\right), 2\left(\begin{array}{c}
n \\
n-1
\end{array}\right)=2 n, \\
& \text { III }\left\langle c_{n k}\right\rangle_{k=0}^{n-1}: 2 n, 2^{2}\left(\begin{array}{l}
n \\
2
\end{array}\right), \ldots, 2^{n-1}\left(\begin{array}{c}
n \\
n-1
\end{array}\right), 2^{n}\left(\begin{array}{l}
n \\
n
\end{array}\right)=2^{n} .
\end{aligned}
$$

Using the algebraic relations $[n ; k]=[n ; n-k]$, for $0 \leqslant k \leqslant n$ and $a_{n k}=$ $c_{n, n-k-1}, c_{n k}=a_{n, n-k-1}$, for $0 \leqslant k \leqslant n-1$, we see that for $n \geqslant 3$ these sequences are first strictly increasing and then strictly decreasing; moreover, II and III have the same elements, but in reverse order. If we replace the number $n$ by an infinite isol $N$, we may or may not permit $k$ to assume values of the type $N-i$, where $i \in \varepsilon$. We obtain

$$
\begin{aligned}
& I^{\prime} \quad\left(\begin{array}{c}
N \\
0
\end{array}\right),\left(\begin{array}{c}
N \\
1
\end{array}\right), \ldots, \quad \quad I^{\prime \prime} \quad\left(\begin{array}{c}
N \\
0
\end{array}\right),\left(\begin{array}{c}
N \\
1
\end{array}\right), \ldots,\left(\begin{array}{c}
N \\
N-1
\end{array}\right),\left(\begin{array}{l}
N \\
N
\end{array}\right), \\
& \text { II' } \quad 2^{N}, 2^{N-1}\left(\begin{array}{c}
N \\
1
\end{array}\right), \ldots, \quad \text { II }^{\prime \prime} \quad 2^{N}, 2^{N-1}\left(\begin{array}{c}
N \\
1
\end{array}\right), \ldots, 2^{2}\left(\begin{array}{c}
N \\
N-2
\end{array}\right), \\
& 2\left(\begin{array}{c}
N \\
N-1
\end{array}\right)=2 N \text {, } \\
& \text { III' }^{\prime} \quad 2 N, 2^{2}\left(\begin{array}{c}
N \\
2
\end{array}\right), \ldots, \quad \quad \text { III }^{\prime \prime} \quad 2 N, 2^{2}\left(\begin{array}{c}
N \\
2
\end{array}\right), \ldots, 2^{N-1}\left(\begin{array}{c}
N \\
N-1
\end{array}\right) \text {, } \\
& 2^{N}\left(\begin{array}{l}
N \\
N
\end{array}\right)=2^{N}
\end{aligned}
$$

Proposition B4.1. For an infinite isol $N$, sequences I', II', III' are strictly increasing. Moreover, sequences $\mathrm{II}^{\prime}$ and $\mathrm{III}^{\prime}$ have no elements in common.

Proof. Define for $A \in \Lambda, P(A, 0)=1$ and

$$
P(A, k)=A \cdot(A-1) \cdot \cdots \cdot(A-k+1), \text { for } 1 \leqslant k \leqslant A ;
$$

$$
P(A, k+1)=(A-k) P(A, k), \text { for } k+1 \leqslant A,
$$




$$
\begin{gathered}
P(A, k)=k !\left(\begin{array}{l}
A \\
k
\end{array}\right), \quad \text { for } k \leqslant A, \\
m P(A, k)<P(A, k+1), \quad \text { for } m, k \in \varepsilon, A \in \Lambda-\varepsilon .
\end{gathered}
$$

Relation (4.3) holds by Theorem 113 of Dekker and Myhill (1960). For $k \in \varepsilon$, $N \in \Lambda-\varepsilon$ we have

$$
\begin{gathered}
\left(\begin{array}{c}
N \\
k
\end{array}\right)<\left(\begin{array}{c}
N \\
k+1
\end{array}\right), \\
2^{N-k}\left(\begin{array}{l}
N \\
k
\end{array}\right)<2^{N-k-1}\left(\begin{array}{c}
N \\
k+1
\end{array}\right), \quad 2^{k}\left(\begin{array}{l}
N \\
k
\end{array}\right)<2^{k+1}\left(\begin{array}{c}
N \\
k+1
\end{array}\right)
\end{gathered}
$$

and the desired statements can be proved from $(4.1), \ldots,(4.5)$.

We also have

PROPOSITION B4.2. For an infinite isol $N$ the tail of $\mathrm{I}^{\prime \prime}$ is the reverse of the head of $\mathrm{I}^{\prime \prime}$. Moreover, the tail of $\mathrm{II}^{\prime \prime}$ is the reverse of the head of $\mathrm{III}^{\prime \prime}$, while the head of $\mathrm{II}^{\prime \prime}$ is the reverse of the tail of $\mathrm{III}^{\prime \prime}$. Finally, I", II", III" are first strictly increasing and then strictly decreasing.

\section{Duality}

Let the word " $n$-polytope" be used the sense of a bounded, convex, $n$-dimensional polytope. For an $n$-polytope $P$ we write $F(P)$ for the class of all its faces and $f_{n k}(P)$ for the number of its $k$-dimensional faces, for $0 \leqslant k \leqslant n-1$. If $P$ and $P^{*}$ are $n$-polytopes, a duality-mapping from $F(P)$ onto $F\left(P^{*}\right)$ is a one-to-one mapping $D$ such that both $D$ and $D^{-1}$ are inclusion-reversing, that is, such that

$$
\alpha \subset \beta \Leftrightarrow D(\beta) \subset D(\alpha), \text { for } \alpha, \beta \in F(P) .
$$

$P$ and $P^{*}$ are $d u a l$, if there exists a duality-mapping from $F(P)$ onto $F\left(P^{*}\right)$; see page 46 of Grünbaum (1967). Note that (5.1) implies: $\operatorname{dim} \alpha+\operatorname{dim} D(\alpha)=n-1$, for $\alpha \in F(P)$. Thus $\operatorname{dim} \alpha=k$ if and only if $\operatorname{dim} D(\alpha)=n-k-1$, hence $f_{n, k}(P)=f_{n, n-k-1}\left(P^{*}\right)$, for $0 \leqslant k \leqslant n-1$; the sequences $\left\langle f_{n 0}(P), \ldots, f_{n, n-1}(P)\right\rangle$ and $\left\langle f_{n 0}\left(P^{*}\right), \ldots, f_{n, n-1}\left(P^{*}\right)\right\rangle$ have therefore the same elements, but in reverse order

Let $Q^{N}=\left\langle 2^{\nu}, F_{\nu}\right\rangle$ and $\mathrm{Oc}^{N}=\left\langle\mu, C_{\mu}\right\rangle$, where $N \geqslant 2$. It is well-known that $Q^{N}$ and $\mathrm{Oc}^{N}$ are dual, if $N$ is finite, say $N=n$. Then the relation $f_{n k}(P)=f_{n, n-k-1}\left(P^{*}\right)$ becomes $a_{n k}=c_{n, n-k-1}$, for $0 \leqslant k \leqslant n-1$, so that $\left\langle a_{n 0}, \ldots, a_{n, n-1}\right\rangle$ and $\left\langle c_{n 0}, \ldots, c_{n, n-1}\right\rangle$ have the same elements, but in reverse order. Now assume that 
$N$ is an infinite isol. Are $Q^{N}$ and $\mathrm{Oc}^{N}$ effectively dual under a suitable generalization of the notion of duality? More specifically, if $\nu$ is immune, $\mu=\nu_{0} \cup \nu_{1}$, $Q^{\nu}=\left\langle 2^{\nu}, F_{\nu}\right\rangle$ and $\mathrm{Oc}^{\nu}=\left\langle\mu, C_{\mu}\right\rangle$, is there a duality-mapping $D$ from $F_{\nu}$ onto $C_{\mu}$ such that the corresponding one-to-one mapping $d$ from $\alpha_{\nu}$ onto $\gamma_{\mu}$ has a partial recursive one-to-one extension and maps the sets in $\left\langle\alpha_{\nu 0}, \alpha_{\nu 1}, \ldots\right\rangle$ onto the sets in $\left\langle\gamma_{\nu 0}, \gamma_{\nu 1}, \ldots\right\rangle$ ? The answer is clearly negative, since $\left\langle A_{N 0}, A_{N 1}, \ldots\right\rangle$ and $\left\langle C_{N 0}, C_{N 1}, \ldots\right\rangle$ have no elements in common for $N \in \Lambda-\varepsilon$ by B4.1. However, II" and III" have the same elements, but in reverse order by B4.2. This suggests that while $Q^{\nu}$ is not effectively dual to $\mathrm{Oc}^{\nu}$, if $\nu$ is immune, there is a system closely related to $Q^{\nu}=\left\langle 2^{\nu}, F_{\nu}\right\rangle$ which might be dual to $\mathrm{Oc}^{\nu}$, namely the system consisting of $2^{\nu}$ and a class of subsets of $\nu$ which behave like faces, but have $\omega$-dimensions of type $N-k$, for $k \geqslant 0$.

Let $N=\operatorname{Req} \nu$ and $N \geqslant 2$. We define a coface of $Q^{\nu}$ as a subset $\tau$ of $2^{\nu}$ for which there exist disjoint subsets $\beta$ and $\gamma$ of $\nu$ such that $\beta$ is infinite, $\gamma$ is cofinite relative to $\nu$ and $\tau=\left\{x \in 2^{\nu} \mid \beta \subset \rho_{x} \subset \beta \cup \gamma\right\}$. We call Req $\gamma$ the $\omega$-dimension of $\tau$, written $\operatorname{dim}_{\omega} \tau$. A coface of $\omega$-dimension $N-k$ is called an $(N-k)$-coface of $Q^{\nu}$. Clearly, $\tau \simeq\left\{x \in 2^{\nu} \mid o \subset \rho_{x} \subset \gamma\right\}$, that is, $\tau \simeq 2^{\gamma}$. Thus each $(N-k)$-coface of $Q^{v}$ has RET $2^{N-k}$. Note that $\tau$ is a coface of $Q^{v}$ if and only if there exist finite subsets $\beta$ and $\delta$ of $\nu$ such that $\beta \cap(\nu-\delta)=o$, that is, $\beta \subset \delta$ and $\tau=\left\{x \in 2^{\nu} \mid \beta\right.$ $\left.\subset \rho_{x} \subset \beta \cup(\nu-\delta)\right\}$. The $G$-number of the coface $\tau$ is the number $j(p, s)$ such that

$$
\tau=\left\{x \in 2^{\nu} \mid \rho_{p} \subset \rho_{x} \subset \rho_{p} \cup\left(\nu-\rho_{s}\right)\right\}, \quad \text { where } \rho_{p} \subset \rho_{s} \subset \nu .
$$

Let $N=\operatorname{Req} \nu$ and $k \leqslant N$. Recall that we write $F_{\nu k}$ for the class of all $k$-faces of $Q^{\nu}$ and $F_{\nu}$ for the class of all faces of $Q^{\nu}$. Similarly, we write $L_{\nu, N-k}$ for the class of all $(N-k)$-cofaces of $Q^{\nu}$ and $L_{\nu}$ for the class of all cofaces of $Q^{\nu}$. It is readily seen that the classes $F_{\nu}$ and $L_{\nu}$ are equal if and only if $\nu$ is finite; they are disjoint if and only if $\nu$ is infinite, for then $F_{\nu}$ consists of finite sets and $L_{\nu}$ of infinite sets. Let $\nu \in N, k \leqslant N, N \in \Lambda_{0}$. Then $\lambda_{\nu, N-k}$ stands for the set of all $G$-numbers of $(N-k)$-cofaces of $Q^{\nu}$ and $\lambda_{\nu}$ for the set of all $G$-numbers of cofaces of $Q^{\nu}$. In symbols,

$$
\begin{gathered}
\lambda_{\nu, N-k}=\left\{j(p, s) \in \varepsilon \mid \rho_{p} \subset \rho_{s} \subset \nu \& r_{s}=k\right\}, \\
\lambda_{\nu}=\left\{j(p, s) \in \varepsilon \mid \rho_{p} \subset \rho_{s} \subset \nu\right\} .
\end{gathered}
$$

Let $\alpha \in N, N \in \Lambda_{0}, k \leqslant N$. Then $\alpha \simeq \beta$ implies $\lambda_{\alpha, N-k} \simeq \lambda_{\beta, N-k}$ and $\lambda_{\alpha} \simeq \lambda_{\beta}$. This enables us to define $L_{N, N-k}=\operatorname{Req} \lambda_{\nu, N-k}$ and $L_{N}=\operatorname{Req} \lambda_{\nu}$, for any $\nu \in N$.

In the remainder of this section it is essential that we distinguish between the $G$-number $G(\tau)$ of some coface $\tau$ of $Q^{\nu}$ and the $G$-number $G(\sigma)$ of some face $\sigma$ of $\mathrm{Oc}^{\nu}$. To stress this distinction we shall henceforth write $G^{\prime}(\sigma)$ rather than $G(\sigma)$ for the $G$-number of a face $\sigma$ of $\mathrm{Oc}^{\nu}$. 
Definition. Let $N \in \Lambda, N \geqslant 2, Q^{\nu}=\left\langle 2^{\nu}, F_{\nu}\right\rangle, \mathrm{Oc}_{f}=\left\langle\mu, C_{\mu}\right\rangle$. Then an effective duality-mapping from $L_{\nu}$ onto $C_{\mu}$ is a one-to-one mapping $D$ from $L_{\nu}$ onto $C_{\mu}$ such that (a) $\alpha \subset \beta$ if and only if $D(\beta) \subset D(\alpha)$, for $\alpha, \beta \in L_{\nu}$, (b) $D\left(L_{\nu, N-k}\right)=$ $C_{\mu, k-1}$, for $0 \leqslant k \leqslant N$, (c) the one-to-one function $d$ from $\lambda_{\nu}$ onto $\gamma_{\mu}$ such that $d G(\tau)=G^{\prime} D(\tau)$ has a partial recursive one-to-one extension.

For an $N$-cube $Q^{\nu}$ with $N \in \Lambda, N \geqslant 2$ we define a facet of $Q^{\nu}$ as an $(N-1)$-coface of $Q^{\nu}$ [hence as an $(N-1)$-face of $Q^{\nu}$ if and only if $N$ is finite]. Recall that in $E^{3}$ we can with a solid cube $Q$ associate its dual, namely a solid octahedron Oc by defining the vertices of Oc as the midpoints of the faces of $Q$ and the edges of $\mathrm{Oc}$ as the line segments which join the midpoints of nonparallel faces of $Q$. We generalize this procedure in the proof of the next theorem, but we replace the midpoints of the facets of the cube by the $G$-numbers of these facets, since we are working with discrete cubes and discrete octahedra.

Proposition B5.1. Let $N \in \Lambda$ and $N \geqslant 2$. Then we can associate with every $N$-cube $Q^{\nu}=\left\langle 2^{\nu}, F_{\nu}\right\rangle$ an $N$-octahedron $\mathrm{Oc}_{f}=\left\langle\mu, C_{\mu}\right\rangle$ and an effective duality-mapping from $L_{\nu}$ onto $C_{\mu}$.

Proof. Assume the hypothesis, $k \geqslant 0$ and write $\nu_{u}$ for $\nu-(u)$, if $u \in \nu$. Recall that every $(N-k)$-coface of $Q^{\nu}$ has RET $2^{N-k}$. The following three statements can now be proved:

(A) Let $\tau$ be a facet of $Q^{\nu}$ and $G(\tau)=j(p, s)$. Then $\tau$ is of one of the two types:

(I) $\tau=\left\{x \in 2^{\nu} \mid(u) \subset \rho_{x} \subset(u) \cup \nu_{u}\right\}$, for some $u \in \nu$.

(II) $\tau=\left\{x \in 2^{\nu} \mid \sigma \subset \rho_{x} \subset o \cup \nu_{u}\right\}$, for some $u \in \nu$.

In fact, $\tau$ is of type (I) if and only if $r_{p}=1 \& r_{s}=1$, while $\tau$ is of type (II) if and only if $r_{p}=0 \& r_{s}=1$.

(B) For each facet $\tau$ of $Q^{\nu}$ there exists exactly one facet of $Q^{\nu}$ disjoint from it, namely $2^{\nu}-\tau$ [we call two facets of $Q^{\nu}$ opposite, if they are complementary subsets of $2^{\nu}$, otherwise adjacent ].

(C) Let $k \geqslant 2$ and $\tau_{1}, \ldots, \tau_{k}$ be $k$ mutually adjacent facets of $Q^{\nu}$ and $\tau=\tau_{1}$ $\cap \cdots \cap \tau_{k}$. Then $\tau$ is an $(N-k)$-coface of $Q^{\nu}$ and $G(\tau)$ can be computed from $G\left(\tau_{1}\right), \ldots, G\left(\tau_{k}\right)$. Moreover, for every $(N-k)$-coface of $Q^{\nu}$ there exists exactly one class $\left(\tau_{1}, \ldots, \tau_{k}\right)$ of $k$ mutually adjacent facets of $Q^{\nu}$ such that $\tau=\tau_{1}$ $\cap \cdots \cap \tau_{k}$.

Using (A), (B) and (C) we now finish the proof. Write $\mu$ for the set of all $G$-numbers of the facets of $Q^{\nu}$; call two elements of $\mu$ opposite (adjacent), if they are $G$-numbers of opposite (adjacent) facets of $Q^{\nu}$. Let $f$ be the iwfp of $\mu$ which maps each element of $\mu$ onto its opposite. Put $\mu_{1}=\{G(\tau) \in \mu \mid \tau$ is of type (I) $\}$, $\mu_{2}=\{G(\tau) \in \mu \mid \tau$ is of type II $\}$. According to (A) we have $\mu_{1}=\left\{j\left(2^{t}, 2^{t}\right) \mid t \in \nu\right\}$ 
and $\mu_{2}=\left\{j\left(0,2^{t}\right) \mid t \in \nu\right\}$, so that $\mu=\mu_{1} \cup \mu_{2}$, and $\mu_{1} \mid \mu_{2}$. Then the function $f$ which maps $f\left(2^{t}, 2^{t}\right)$ and $j\left(0,2^{t}\right)$ onto each other, for $t \in \nu$, has a partial recursive one-to-one extension. Hence $f \in \operatorname{Inv}_{\omega}(\mu), \mu_{1} \simeq \mu_{2} \simeq \nu$ and $\operatorname{Req} \mu=2 N$. We now show that $\mathrm{Oc}_{f}=\left\langle\mu, C_{\mu}\right\rangle$ satisfies the requirements. Note that $\operatorname{dim}_{\omega} \mathrm{Oc}_{f}=N$, since Req $\mu=2 N$. Define for $k \geqslant 2$ the mappings $D_{k}$ and $d_{k}$ by:

$$
\begin{gathered}
\operatorname{Dom} D_{k}=L_{\nu, N-k}, \quad D_{k}\left(\tau_{1} \cap \cdots \cap \tau_{k}\right)=\left(G\left(\tau_{1}\right), \ldots, G\left(\tau_{k}\right)\right), \\
\delta d_{k}=\lambda_{\nu, N-k}, \quad d_{k} G\left(\tau_{1} \cap \cdots \cap \tau_{k}\right)=G^{\prime}\left(G\left(\tau_{1}\right), \ldots, G\left(\tau_{k}\right)\right) .
\end{gathered}
$$

for any $k$ mutually adjacent facets $\tau_{1}, \ldots, \tau_{k}$ of $Q^{\nu}$. By (C) the mapping $D_{k}$ maps $L_{\nu, N-k}$ one-to-one onto $C_{\mu, k-1}$ so that $d_{k}$ maps $\lambda_{\nu, N-k}$ one-to-one onto $\gamma_{\mu, k-1}$. Moreover, $d_{k}$ and $d_{k}^{-1}$ have partial recursive extensions, hence $d_{k}$ has a partial recursive one-to-one extension. Define the mappings $D$ and $d$ as follows: for $\tau \in L_{\nu}, x=G(\tau)$ and $\operatorname{dim}_{\omega} \tau=N-k$,

$$
\begin{array}{ll}
D(\tau)=o, & d(x)=G^{\prime}(o), \text { for } k=0, \text { that is, } \tau=2^{v}, \\
D(\tau)=(G(\tau)), & d(x)=G^{\prime} \text { of }(x), \text { for } k=1, \text { that is, } \tau \text { is a facet, } \\
D(\tau)=\left(G\left(\tau_{1}\right), \ldots, G\left(\tau_{k}\right)\right), & d(x)=G^{\prime}\left(G\left(\tau_{1}\right), \ldots, G\left(\tau_{k}\right)\right), \text { for } k \geqslant 2,
\end{array}
$$

where in case $k \geqslant 2, \tau_{1}, \ldots, \tau_{k}$ are the $k$ mutually adjacent facets of $Q^{v}$ such that $\tau=\tau_{1} \cap \cdots \cap \tau_{k}$. Given an element $x=j(p, s) \in \lambda_{\nu}$, we can compute $k=r_{s}$ and $d(x)=d_{k} j(p, s)$. Thus the one-to-one function $d$ from $\lambda_{\nu}$ onto $\gamma_{\mu}$ has a partial recursive one-to-one extension. Also, $D\left(L_{\nu, N-k}\right)=D_{k}\left(L_{\nu, N-k}\right)=C_{\mu, k-1}$, hence $d\left(\lambda_{\nu, N-k}\right)=\gamma_{\mu, k-1}$, for $k \geqslant 0$. Let $\left(\tau_{1}, \ldots, \tau_{k}\right)$ and $\left(\tau_{1}^{*}, \ldots, \tau_{m}^{*}\right)$ be classes of mutually adjacent facets of $Q^{\nu}$ with $\tau_{1} \cap \cdots \cap \tau_{k}=\tau$ and $\tau_{1}^{*} \cap \cdots \cap \tau_{m}^{*}=\tau^{*}$. Then $\tau \subset \tau^{*}$ if and only if $\left(\tau_{1}^{*}, \ldots, \tau_{m}^{*}\right) \subset\left(\tau_{1}, \ldots, \tau_{k}\right)$ if and only if $\left(G\left(\tau_{1}^{*}\right), \ldots, G\left(\tau_{m}^{*}\right)\right) \subset\left(G\left(\tau_{1}\right), \ldots, G\left(\tau_{k}\right)\right)$ if and only if $D\left(\tau^{*}\right) \subset D(\tau)$.

COROLLARY. $L_{N, N-k}=2^{k}\left(\begin{array}{c}N \\ k\end{array}\right)$ and $L_{N}=3^{N}$, for $N \in \Lambda_{0}, 0 \leqslant k \leqslant N$.

Remark (A). Let $Q^{\nu}=\left\langle 2^{\nu}, F_{\nu}\right\rangle, \mathrm{Oc}^{\nu}=\left\langle\mu, C_{\mu}\right\rangle, N=\operatorname{Req} \nu, N \in \Lambda, N \geqslant 2$. Write $C Q^{\nu}$ for $\left\langle 2^{\nu}, L_{\nu}\right\rangle$ and call $C Q^{\nu}$ effectively dual to $\mathrm{Oc}^{\nu}$, if there is an effective duality-mapping from $L_{\nu}$ onto $C_{\mu}$. Then we have

(I) If $N$ is infinite, $Q^{\nu}$ is not effectively dual to $\mathrm{Oc}^{\nu}$,

(II) $C Q^{v}$ is effectively dual to $\mathrm{Oc}^{\nu}$, for every $N$ (finite or infinite); however, for a finite $N$ this only yields the well-known fact that the $n$-cube is dual to the $n$-octahedron, for in that case $L_{v}=F_{v}$, hence $C Q^{\nu}=Q^{\nu}$.

REMARK (B). Under the hypothesis of the preceding remark it is also possible to define a class $H_{\mu}$ of cofaces of $\mathrm{Oc}^{\nu}=\left\langle\mu, C_{\mu}\right\rangle$ such that there exists an effective duality-mapping from $F_{\nu}$ onto $H_{\mu}$. Thus the systems $\left\langle 2^{\nu}, F_{\nu}, L_{\nu}\right\rangle$ and $\left\langle\mu, C_{\mu}, H_{\mu}\right\rangle$ are effectively dual in the sense that there is not only an effective duality-mapping 
from $L_{\nu}$ onto $C_{\mu}$, but also one from $F_{\nu}$ onto $H_{\mu}$. If $N=\operatorname{Req} \nu$ is finite, we have $F_{\nu}=C_{\mu}$ and $L_{\nu}=H_{\mu}$ and we can identify $\left\langle 2^{\nu}, F_{\nu}, L_{\nu}\right\rangle$ with $Q^{\nu}=\left\langle 2^{\nu}, F_{\nu}\right\rangle$ and $\left\langle\mu, C_{\mu}, H_{\mu}\right\rangle$ with $\mathrm{Oc}^{\nu}=\left\langle\mu, C_{\mu}\right\rangle$.

\section{References}

J. N. Crossley and A. Nerode (1974), Combinatorial functors (Springer-Verlag, New York).

J. C. E. Dekker and J. Myhill (1960), 'Recursive equivalence types', University of California Publications in Mathematics (N.S.) 3,67-214.

J. C. E. Dekker (1981), 'Recursive equivalence types and cubes', Aspects of effective algebra, ed. J. N. Crossley, pp. 87-121. (Upside Down A Book Company, Yarra Glen, Victoria, Australia).

J. C. E. Dekker (1981a), 'Twilight graphs', J. Symbolic Logic 46, 539-571.

R. M. Friedberg (1961), 'The uniqueness of finite division for recursive equivalence types', Math. $Z$. 75, 3-7.

B. Grünbaum (1967), Convex polytopes (Interscience Publishers, New York).

M. Jungerman and G. Ringel (1978), 'The genus of the $n$-octahedron: regular cases', J. Graph Theory 2,69-75.

A. Nerode (1961), 'Extensions to isols', Annals of Mathematics 73, 362-403.

J. B. Remmel (1981), 'Effective structures not contained in recursively enumerable structures', Aspects of effective algebra, ed. J. N. Crossley, pp. 206-225 (Upside Down A Book Company, Yarra Glen, Victoria, Australia).

The Institute for Advanced Study

Princeton, New Jersey 08540

Rutgers University

U.S.A.

New Brunswick, New Jersey 08903

U.S.A. 